\title{
A case study of patient journey mapping to identify gaps in healthcare: Learning from experience with cancer diagnosis and treatment
}

\author{
Andre W. Kushniruk \\ Elizabeth M. Borycki \\ University of Victoria, Victoria, BC, Canada \\ Avi Parush
}

Technion - Israel Institute of Technology, Technion City, Haifa, Israel

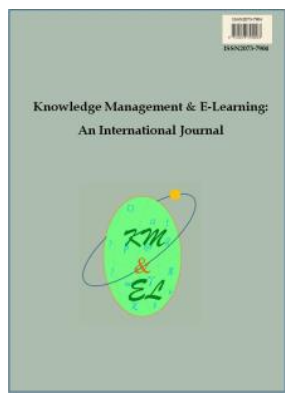

Knowledge Management \& E-Learning: An International Journal (KM\&EL) ISSN 2073-7904

Recommended citation:

Kushniruk, A. W., Borycki, E. M., \& Parush, A. (2020). A case study of patient journey mapping to identify gaps in healthcare: Learning from experience with cancer diagnosis and treatment. Knowledge Management
\&
E-Learning,
$12(4)$
405-418.

https://doi.org/10.34105/j.kmel.2020.12.022 


\title{
A case study of patient journey mapping to identify gaps in healthcare: Learning from experience with cancer diagnosis and treatment
}

\author{
Andre W. Kushniruk* \\ School of Health Information Science \\ University of Victoria, Victoria, BC, Canada \\ E-mail: andrek@uvic.ca
}

\section{Elizabeth M. Borycki}

School of Health Information Science

University of Victoria, Victoria, BC, Canada

E-mail: emb@uvic.ca

\section{Avi Parush}

Faculty of Industrial Engineering and Management

Technion - Israel Institute of Technology, Technion City, Haifa, Israel

E-mail: aparush@technion.ac.il

\section{*Corresponding author}

\begin{abstract}
Journey mapping methods have a number of practical uses. One of the most promising applications in the area of healthcare is to apply patient journey mapping to identify a patient's pathway through their healthcare journey. Nowhere is this more important than in the area of cancer care. With lengthy wait times in many countries and the complexity of care paths that cancer patients travel, there is ample opportunity to identify both gaps in care as well as opportunities to improve care processes. In this article the authors discuss a case study of a patient journey involving multiple care organizations, several health professionals and care in both Canada and the United States. By applying patient journey mapping a simplified version of such complexity can be presented in a visual and succinct way, allowing health professionals and managers of healthcare organizations to identify where inefficiencies in care and patient safety issues occur. Furthermore, this mapping can form the basis for optimizing care processes and holds considerable promise for patientcentred healthcare. Implications of using patient journey mapping for improving cancer care and healthcare more generally are discussed.
\end{abstract}

Keywords: Patient journey mapping; User journey mapping; Human factors; Health information; Consumer e-Health; Healthcare

Biographical notes: Dr. Andre Kushniruk is Professor and Director of the School of Health Information Science at the University of Victoria, British Columbia, Canada. He has published widely in the area of health informatics and is known for his work in the usability of healthcare information systems. Dr. Kushniruk conducts research in a number of areas and he focuses on developing new methods for the evaluation of information technology in 
healthcare. Dr. Kushniruk has been a key researcher on a number of national and international collaborative projects. His work includes evaluation of systems for use by healthcare providers, patients and citizens.

Dr. Elizabeth Borycki is a Professor in the School of Health Information Science at the University of Victoria, Canada. She is the Director of the Global Laboratory for Digital Health Innovation at the University of Victoria, Canada. Elizabeth conducts research at the intersection of safety science, human factors and technology in the field of health informatics. She is also a Clinician Scientist with the Michael Smith Foundation for Health Research, Vancouver, Canada. Elizabeth's research interests include safe health technology design, software engineering and implementation science involving health technology.

Dr. Avi Parush is a Professor at the Industrial Management and Engineering Faculty, The Israel Institute of Technology, and an emeritus professor at Carleton University, Ottawa, Canada. He received his $\mathrm{PhD}$ in Psychology from McGill University, Montreal, Canada. His current research focuses on healthcare with a particular emphasis on how HCI and usability influence patient experience, safety, and empowerment. He is the lead author of the book: "Human Factors in Healthcare: A Field Guide to Continuous Improvement", he is the founding editor in chief of the Journal of Usability Studies, and is currently a section editor in the Journal of Medical Internet Research - Human Factors, and on the editorial board of the Human Factors journal.

\section{Introduction}

Patient journey mapping holds considerable promise as an instrument that can be used to help visualize the processes of healthcare from a patient perspective over time. One of the most important applications of journey mapping is the area of improving healthcare activities, including the integration of health information technology into the process of care. By focusing on the patient journey, such mapping could identify gaps in healthcare processes (e.g. long wait times) and suboptimal processes (e.g. impact of missed or incorrect diagnoses). It could also identify areas where improvements could be made to improve the overall journey and ultimately patient outcomes. Along these lines Parush, Levin, and Campbell (2014) have described an approach known as dual patienthealthcare provider experience mapping and have discussed the implications for information technology deployment. This approach integrates consideration of experiences of both patients and the healthcare system in mapping the patient journey (Parush et al., 2014; Parush, 2019). By focussing on multiple perspectives and illustrating a journey along multiple timelines representing different aspects of a journey, this approach can allow for identification of gaps in healthcare processes that could be mitigated.

In this paper a patient-specific case study is presented. It is a personal patient journey that involved a complex set of organizations, information systems and decisionmaking processes that spanned two countries, multiple healthcare contexts and the use of an array of information sources available to patients on the Internet across the WWW. Patient journey mapping will be applied to visualize and simplify the journey to help in identifying where issues in healthcare processes can be identified and rectified. The case study will also emphasize the need to encourage patient empowerment and patient input into decision making through the use of varied health information sources, systems, devices and tools (Eysenbach \& Kohler, 2002). 


\section{A personal case study: Patient journey mapping in cancer care and the role of health information}

Patient journey maps can be used to identify where gaps in healthcare processes exist. They can be used to indicate how health information and health information systems could play an important role in filling those gaps. To illustrate this, a case study will be presented based on the lived experiences and patient journey of one of the authors - AK (Andre Kushniruk). The case description in terms of patient mapping highlights issues related to availability of information for patients, as well as opportunities for applying patient journey mapping to identify solutions to problems in healthcare in order to lead to the best possible health outcomes. To illustrate the importance of health information for patients, the journey through multiple health systems and contexts will be described in terms of several phases over time (as is typical with patient journey mapping) alongside a discussion of the use of health information technologies, information systems and information gaps encountered along the way.

\subsection{Phase 1 - Initial stages from symptoms to diagnosis}

The author's (AK) patient journey began with the first symptoms of illness in the late spring and early summer of 2017. The initial symptoms consisted of low-grade pain when eating, swallowing and speaking. After visiting two dentists late that summer both dentists concluded it was a dental issue and not serious as I had never been a smoker and I did not have risk factors for cancer. Both dentists took photos of the affected regions and indicated they would track it, and monitor it, without further referring me to a physician. However, as symptoms of pain persisted and worsened during the summer of 2017 the need to seek the advice of a general practitioner (GP) became apparent to me.

At the time of the first appointment with the GP it was clear there was something more serious behind the symptoms and the symptoms could be indicative of a larger issue. As a consequence, my GP referred me to an ear nose and throat (ENT) specialist. The phase of waiting for the appointment with the local ENT specialist then began and despite my condition being considered urgent, it took over a month before the ENT would eventually see me, in early October.

At the appointment, the ENT indicated that I may have tongue cancer. This led to a series of tests over the next month, beginning with a biopsy, then magnetic resonance imaging (MRI) two weeks later in October, followed by a positron emission tomography (PET)/computed tomography (CT) scan several weeks after that (in November). The test confirmed an initial diagnosis of tongue cancer. This was shocking news for me, but not as shocking as when two weeks later the same ENT stated (based on the MRI scan) that not only did I have a tumor, but that it was now too large to operate on. The ENT indicated that surgery would no longer be possible, as it would not result in obtaining "clear tumor margins" and so would not be successful from his perspective. As a result, I would not be receiving life-saving surgery. When I probed for further details as to my chances of survival, the ENT indicated that it was going to be poor and when further probed he indicated I could be considered to be palliative. I was then scheduled for radiation and chemotherapy treatment, to start approximately one and half months later. My symptoms worsened quickly over this waiting period and I was finding it difficult to eat and speak.

From an emotional perspective, during this phase I felt stunned by the turn of events and focussed on considering the impact of what I surmised as my likely death would be on my friends and family. However, at this point my wife (a health information 
specialist and nurse) began to check the WWW for cases like mine to see if a better outcome could be achieved. After consulting the WWW (initially involving searching Google for information), other considerations began to come to mind. This included wondering why I could not have received surgery, as a follow-up PET/CT scan that came later did not indicate that the cancer had spread to other parts of my body (and according to web resources the survival outcomes should be better with surgery). During this time, to compound the situation I was beginning to have episodes where I had bouts of excessive bleeding from the tumor area, leading to several emergency room visits, with no resolution of that issue.

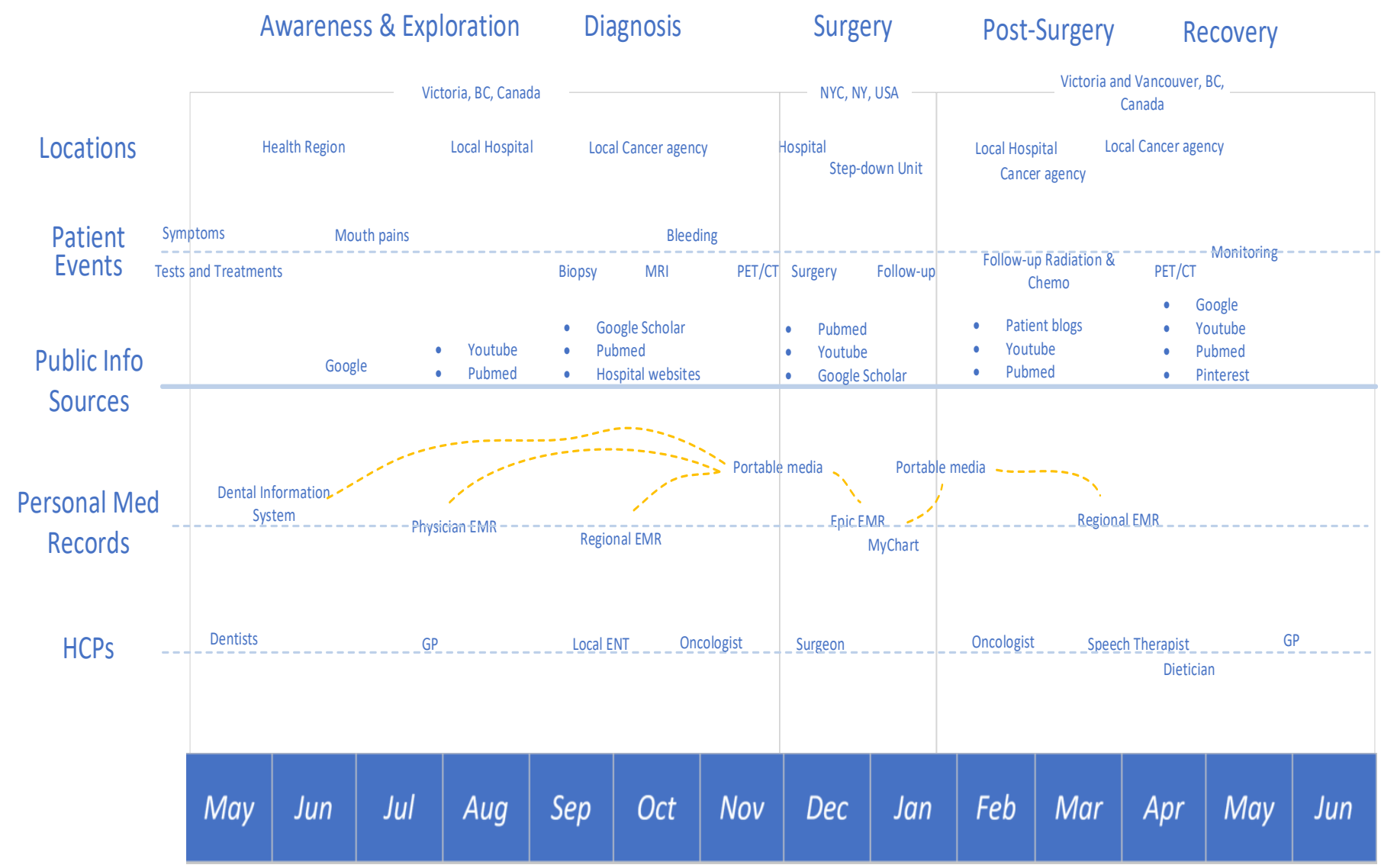

2017

Fig. 1. Patient journey map for the cancer case study

Fig. 1 shows the patient journey map corresponding to the evolution of my case, with the months in duration of events indicated along the bottom of the figure. The horizontal time lines in the figure (starting from the top of the figure) correspond to the following: (1) the physical locations where events occur to the patient - labelled as "Locations", (2) what happened from a patient perspective - "Patient Events", (2) what publically available information resources were accessed - "Public Info Sources", (3) what electronic health records and systems were involved in the case - "Personal Med Records" and (4) the healthcare professionals involved over time - "HCPs". In addition, 
the phases of the journey are indicated and labelled across the top of the figure: (1) "Awareness and Exploration", (2) "Diagnosis", (3) "Surgery", (4) "Post-Surgery", and (5) "Recovery". This type of visualization can succinctly map complex patient issues, personal perspective and healthcare actions and activities along a timeline. For example, the line labelled "Public Info Sources" shows the use of different types of information along the entire journey from symptoms to diagnosis through to surgery and recovery.

\subsubsection{Personal use of the $W W W$}

While waiting for the appointment to see the ENT specialist my spouse and I began using the Internet (using the Google search engine) to examine all possible treatments across the WWW. This included reviewing information from the Canadian and America cancer society websites - see the timeline labelled "Public Info Sources" in Fig. 1. This line shows the range of different public information sources that were personally accessed over time. The searched online sources indicated that if the cancer had not spread past my head and neck region (as it hadn't), then with an appropriate treatment there should be statistically a good chance of surviving (approximately 63\% given the stage of cancer), leading to a possible cure (Canadian Cancer Society, 2020). My personal access to such information over the Internet on the WWW became critical in giving me hope and in seeking further information from the Internet for the next phase of my journey. These resources essentially allowed me to check about my chances of survival. By using the Internet and personally checking blogs and posts from other cancer patients we were able to determine that surgery could possibly lead to a cure. Furthermore, we found from searching that even extensive surgery involving a near total glossectomy (i.e., near total removal of my tongue) would not necessarily result in a low quality of life. In fact, many personal testimonials on the WWW indicated that using the most advanced methods available for removing the tongue and replacing it with tissues from other parts of the body (such as transplanted leg muscle to reconstruct a new tongue) a good quality of life could be achieved, along with a complete cure from cancer.

\subsubsection{Institutional information systems}

In considering the use of technology during this phase of the patient journal, all the medical information about my case was initially located in several different electronic medical record systems (EMRs) in my local region (see the horizontal line in Fig. 1 labelled "Personal Med Records" - the second row from the bottom in the figure, which shows the flow of information through different systems over time). One of these EMRs was used by my GP in his office for recording the information from my visits over time. A second EMR was used in my local health region which contained information including biopsy results from my local hospital. A third EMR was used by my local cancer agency for my cancer care. In addition, the two dentists I had visited before seeing my GP had stored photographs of my mouth in their own dental information systems. However, these dental systems were not connected to any of the other EMRs and that data could not be passed on to the other health professionals in my acute or community care.

\subsubsection{Information gaps}

During this initial phase (corresponding to May to November 2017 in Fig. 1) a number of information gaps became apparent. Firstly, information about my case from the dentists (e.g. photos) were stored in the dentists' local dental systems in their offices and were 
never actually seen by my GP or the acute and cancer care physicians connected to their medical information systems. An exchange of information early on from my dentist could have potentially averted the serious issues that occurred later on by alerting my health professionals of an ongoing and unresolved health issue.

Secondly, I was not initially given information comparing what my statistical chances of survival would be for different treatment options - rather I had to search the Internet for this information on my own. This is critical information for helping patients and their healthcare providers come to the best decisions for the patient's outcome and would have been essential for patient empowerment.

In my case, relevant and timely health information related to my condition was not provided and as a result I sought relevant information from the WWW, specifically information about the " 5 year survival curve" for tongue cancer - a graph that indicates survival statistics for cancer patients receiving different types of treatments. Additionally, through my independent research on the WWW, I was able to find comparable cases of other patients who appeared to face a similar situation but who had received surgery, recovered and were cured. This highlights the need for more accessible information and decision aids for use by patients, and direction of patients to such online sources. It also further underlies the need for patients knowing how to access the "right" information.

\subsection{Phase 2 - Exploring possibilities for life-saving surgery}

From searching reputable publicly available online information sources (shown in Fig. 1) it was clear that undergoing surgery would lead to the possibility for a potential cure (involving surgical removal of the tongue cancer) with a statistically greater than $60 \%$ chance of survival long-term given my condition. These statistics were appealing and potentially applicable to me, as my cancer was considered "regional", as it had not spread distally to other regions of my body. In addition, the online searching confirmed that without the surgery the chances of my five-year survival would have only been in the 20$30 \%$ range (Zhang et al., 2013).

Therefore, my perspective about my diagnosis began to shift from one of hopelessness to one where there was hope that the cancer was operable and even possibly curable. As a result, my spouse and I began to search of the leading articles on the Internet for literature that would guide us in taking tangible actions towards seeking alternative treatment options. Eventually, using the health search engine PubMed, we were able to locate a particular surgeon and hospital where there would have a good chance of getting the operation and ultimately being cured. This would involve getting the life-saving surgery, involving removal of my tongue. The hospital we identified was a major international centre in New York, and coincidentally turned out to be the part of the same organization I had once worked with. Within a few days I was able to get an appointment with one of the world's top surgeons for a consultation. I flew to New York within days and received a rapid second opinion regarding my chances of survival and best treatment path. Thus, the WWW was also used to locate a hospital where I could receive surgery, as well as a expert surgeon, and thus began the next phase of my journey (see the column in Fig. 1 labelled "Surgery").

\subsubsection{Personal use of the $W W W$}

By searching PubMed, Google, YouTube and patient blogs on the Internet (see the horizontal line "Public Info Sources" under the column of the "Diagnosis" phase in Fig. 
1), I was able to determine that what appeared to be a bleak situation could be turned around (corresponding to the period of October - November in Fig. 1). Furthermore, according to the publically available information resources and research publications my spouse and I accessed over the WWW, with the right treatment I should have a good chance for long-term survival. Furthermore, online searching indicated that in other localities life-saving surgery was possible and had been conducted for patient cases very similar to mine. A further search of online resources was conducted by us, including a search of the American and Canadian cancer society websites. This searching also indicated that there was clearly hope that my survival chances should have been at least double (i.e., in the 60-70\% survival range, versus 30\% chance of long-term survival). These Internet searches were instrumental in changing my frame of thought regarding the possibility of having treatment that could lead to a cure.

\subsubsection{Institutional information systems}

In preparing for going to New York for obtaining a second opinion from a renowned surgeon (found over the WWW and PubMed) a wide variety of my personal information had to be collected. This was a very time sensitive as my symptoms were becoming worse by the day and the possibility that the cancer would spread to other parts of my body if any further time went by waiting for treatment. In preparation for my initial trip from Canada to New York, I collected all the information from all the various EMRs in Canada that might be relevant for my case, requesting release of that information from multiple institutions (as the computer systems in New York would not be connected to any of those systems in my home in Canada). This included PDF's and paper print outs of reports about my health history that were stored in the various EMRs of my providers. In addition, I requested to have my scans copied to CDs that I could take across the border for the surgeon in New York to review when I had my appointment with him. Finally, I was able to obtain from my local hospital in Canada the actual slides that were made from my biopsy to bring with me on my upcoming flight to New York and present to the surgeon there for re-assessment.

\subsubsection{Information gaps}

My experience in this phase of my journey indicated a range of information gaps. For one, in my home locality I was not referred to any ENT specialist other than the one I was initially referred to, and there was no suggestion or advice for obtaining a second opinion regarding treatment possibilities (nor was there information about ENTs in my province who could perform this surgery on the WWW). Compounding that, I had tried to locate an alternative ENT specialist in my locality on my own, but this information was not readily available as a patient. Timely access to that information could have helped me in time and alleviated much of the trauma and distress. In contrast, by using the online search engine PubMed it was possible to find names of ENT specialists and locations internationally where I could obtain the life-saving operation I required. However, unfortunately none of those were located in the area I lived in.

Other issues included having to manually print out reports (from all the EMRs used in my region that had information about my case) to take with me, and obtain other information I needed before I flew to New York - essentially manually transporting that information from my health system in Canada to that in another country. Finally, perhaps the biggest gap I faced as a patient was that I had to find information about critical 
aspects of care (such as the five-year survival statistics for different treatment options) on my own.

\subsection{Phase 3 - Life-saving surgery}

From my first visit to the surgeon in New York, we discussed treatment options along with a clear presentation to me of potential risks of operating. My mood changed from feeling depressed about my future to feeling very positive, comfortable and even optimistic. The surgeon in New York told me he could remove the tumour surgically and that he could also remove any cancer associated with its spread to my lymph nodes, with the ultimate intent of a total cure. He also indicated that with the removal of the tumour in his patients had resulted in a five-year survival rate at close to $70 \%$ and there was a good chance of my being completely cured by having the operation. He also indicated that any portions of my tongue that might have to be removed by the surgery would be reconstructed so I should be able to speak and swallow food and continue working. I was both shocked and overjoyed that this would be possible! The surgeon immediately suggested some surgical dates given my condition. I was amazed that he could book me for surgery only a week and a half later, seeing the urgency of my situation. Just before the surgery I needed to return briefly to Canada to make preparations for the operation in New York.

My operation was extensive and took several hours, and involved removal of most of my tongue, and removal of 62 lymph nodes in my neck. The surgery concluded with the creation of a new tongue from muscle taken from my thigh. Despite the extent and intensity of the operation upon waking up in the recovery room I felt overjoyed as the pain I had previously experienced was completely gone (as my surgeon in New York had indicated might happen). I then spent ten days in a special step-down unit (an intermediary care unit where patients' criticality lies between the intensive care unit (ICU) and the general surgical recovery unit) in the hospital in New York. This was a critical period and I was closely monitored and encouraged to try to eat and speak as soon as possible. Every day I was closely monitored to see if all had gone well and my "new" tongue had taken hold. However, at that point in time I was unsure if I would be able to speak again and had some other concerns (as I had initially failed my post-surgery test of swallowing ability). As my recovery improved and time progressed in the hospital in New York it became clear that the operation was a success and I was able to incrementally regain my ability to eat, speak and swallow over the months that followed.

\subsubsection{Personal use of the $W W W$}

While in the hospital in New York my wife and I continued to use the Internet and the WWW to track what was going on with my treatment and operation (see Fig. 1 row 3 "Public Info Sources" for the months of December and January of my patient journey). We were happy and reassured to find that the procedures that were taking place and my care plan were following the latest guidelines and recommendations - many of which actually emerged from the hospital and team of experts who were caring for me in New York. Furthermore, when I first experienced difficulty swallowing immediately after the operation, both my spouse and myself watched YouTube videos devoted to problems with swallowing, patient blogs and other online resources, specifically to help those having problems swallowing and speaking.

The websites and online media we found were very helpful in both improving my condition and my regaining confidence in being able to someday be back to normal. Thus, 
the Internet once again became a critical source of information for planning my next steps for my well-being and surgical recovery. Technological access to pertinent health information also enabled me to become an active participant in my own patient journey. I was also able to ensure that my latest findings about my condition were consistent with the treatment I was receiving there.

\subsubsection{Institutional information systems}

In going to New York for an operation I was essentially moving my healthcare journey to another country, at least temporarily (as I did return to Canada for follow-up radiation and chemotherapy treatment). This meant that information and data about me that was available in Canada was not going to be available in the United States. In preparing for the operation, I had physically carried as much information as I could with me (e.g. patient records, scan reports etc.) which were then scanned into the integrated institutionwide electronic medical record (EMR) system used at the hospital in New York. The system in New York is considered state-of-the-art and also included a web portal designed for direct access over the Internet by patients (essentially a personal health record - PHR) that allows me to directly access a good portion of the information contained in the hospital's EMR (a patent portal known as MyChart). This technology has revolutionized patient access to their own data electronically, and I had once been involved in developing the first such application in the world several decades before (Cimino, Patel, \& Kushniruk, 2002). Upon leaving New York to return back to Canada I once again took a paper print out of my patient records (this time from the New York EMR) with me to bring back to Canada to my local cancer agency - at this point the print out with information about my case was massive, with several hundred pages.

\subsubsection{Information gaps}

Leaving Canada and going to another country for treatment (i.e., the United States) for an operation posed a number of both logistical and informational issues, especially as my time was clearly running out. Fortunately, I was able to collect up the needed information, physical discs with scans and other needed artifacts (e.g. the physical biopsy slides) in time for my trip to New York. However, in the future it would be ideal to have all this information made available for full electronic transfer across borders in such emergency situations, despite issues related to policies regarding transferring health data across borders and the legal-ethical issues associated with that. Having access to a patient portal was useful for accessing key aspects of my own record as was continued access to a range of resources personally available over the WWW (including PubMed, patient blogs etc.). To fill in gaps in information about my case from New York and Canada, before returning to Canada my oncologist in Canada kept close contact with me about my case through phone calls in preparing for my return to Canada for follow-up radiation and chemotherapy using the phone as the main channel for communication. As I was once again moving my information to another system (i.e., back to Canada) I relied on actual paper print-outs of records and reports I had obtained in New York for carrying with me back to Canada.

\subsection{Phase 4-Post-surgery: Follow-up radiation and chemotherapy}

Upon returning to Canada after my operation in New York, I entered yet another extensive phase of my journey (corresponding to the months Jan - March in Fig. 1) consisting of follow-up radiation and chemotherapy. This treatment was intended to 
reduce the chance of the cancer recurring after my operation. In addition, although my operation was successful there were some issues with swallowing and speaking. However, over time I gradually regained improved ability to eat, speak and swallow. I also knew that on my return to Canada I would require seven weeks of intensive follow up radiation and chemotherapy. This follow up (i.e., adjuvant) therapy, which I received once back in Canada during January to March of 2018, consisted of daily trips to my local Cancer Agency where I received targeted radiation to my head and neck over a several week period. Additionally, during the course of my treatment I was admitted to the local hospital for two weekends where I was administered a strong chemotherapy agent in order to further mitigate the risk of the cancer recurring by potentiating the impact of the radiation treatment.

During this part of my journey, I continued with my work full time (as a university professor) but worked at home and did feel tired and at times had further difficulty eating and speaking (which was to be expected during this follow-up cancer treatment). Fortunately, I was able to physically handle the treatment and it proved successful in the long term. As further follow up, every few months I had new scans to ensure the cancer was not recurring.

\subsubsection{Personal use of the $W W W$}

During this phase I relied on the advice from my local Cancer Agency and the information on the WWW to understand what the procedures I would receive involved, including their risks, side effects and potential for improving my outcome. Again, the WWW allowed me to access patient stories, blogs and official healthcare websites that contained information about the experiences of others who had gone through this type of journey before. This quick and timely access to information was not only greatly reassuring but $\mathrm{I}$ also found tips that were useful in helping me get through (e.g. finding the recommendation to drink milkshakes to increase my caloric intake during the worst periods where radiation treatment burned my throat, making eating solid food literally impossible). Moreover, I was able to share what I learned online with local healthcare team and this also greatly facilitated my recovery.

\subsubsection{Institutional information systems}

Being back in Canada for follow-up treatment (using radiation and chemotherapy) much of the information about my case were transferred to the EMRs in my local area. I continue to visit my local GP (who uses an EMR system) and also have regular follow-up appointments at my local cancer agency. In Canada I did receive considerable support for help with eating and speaking from my local cancer agency health professionals. My personal use of publically available Internet resources also allowed for me to delve into details of the process of recovery and also come up with potential solutions to issues (e.g. around eating and speaking) I could then bring to my healthcare providers at my local cancer agency to discuss.

\subsubsection{Information gaps}

There were a number of information gaps as a consequence of my care having involved treatment in two countries (surgery in the US and follow-up chemo and radiation in Canada) with several different healthcare systems and organizations. As my medical reports in the computer systems in New York were not connected to or integrated with 
the ones in Canada I have had to contact the hospital in New York (where I had received my surgery) on several occassions for information relevant to my Canadian healthcare providers.

\subsection{Phase 5 - Long-term recovery}

In this final phase of my journey, the focus of my attention shifted from a curative focus to how to recover from the surgery, chemotherapy and radiation in order to fully regain my ability to eat and speak (corresponding to the months April - June 2018 in Fig. 1). This involved help from a speech therapist and dietician at the Cancer Agency over a several month period from January until June 2018. I wanted to continue to be an empowered active participant in my own care by staying informed of the latest trends and research on my previous condition and therefore I continued to research and read relevant articles online from PubMed and other reputable sources. I also explored blogs and postings on YouTube and Pinterest, posted by patients who had undergone similar treatment and therapy. This aided in not only giving me realistic expectations regarding recovery, but also in sharing strategies and tips for the road to recovery. Once again, the Internet became a big part of my patient journey and helped me to become increasingly confident about my future and long-term health outcome over the ensuing months.

I am now three years cancer free, which according to the statistics indicates I should be cancer free for the long term, now officially having "beat cancer". Furthermore, I have continued with all activities I undertook before becoming ill. Much of what happened and I how I was able to turn around what appeared to be a bleak situation I can attribute to my friends and colleagues, a range of health professionals, and the information we gleaned from the Internet that was critical to my health and well-being.

\subsubsection{Personal use of the $W W W$}

As a result of my experience, I continue to use the WWW extensively to keep with advances in treatment of the cancer I had, to read about others' experiences and to learn more about how to identify any future symptoms that might indicate a recurrence. I also encourage others (through my teaching, patient journey research and work) who may be facing some of the same difficult health scenarios to become familiar with the array of information resources available to all of us. The information I continue to search for from the WWW is an invaluable aid in understanding and discussing long-term monitoring decisions with my health professionals and I continue to personally search for information relevant to cancer care in general and for new advances. In addition, as a health information science researcher I have started several new paths of research to help patients understand the full range of treatment options and present them with effective information visualizations that can be shared between them and their health providers.

\subsubsection{Institutional information systems}

My health information related to this case is now distributed across numerous health information systems (e.g. EMRs in Canada and the US) and organizations (e.g. my local GP, operating surgeon, local cancer agency etc.). In addition, I have become "curator" of my own critical health information, creating a personal database of all the reports, scans and relevant articles I have collected along my journey. I have compiled all this information personally as spreadsheets and files on my own personal computer, and continue to do so. 


\subsubsection{Information gaps}

During my patient journey I have been keenly aware of areas where there are gaps in information and information silos. Some of this comes from having not only system but also organizational gaps that could be solved using technologies to provide and support improved collaboration and joint problem solving between patient and providers. Along these lines, there are many tools and technologies that could support sharing not only of information, but also the decision process itself to allow for true patient empowerment. Use of methods such as patient journey mapping to outline and identify barriers to achieving successful health outcomes will be essential.

\section{Discussion}

As part of the patient journey, I have reflected on many of the things I have learned from the experiences described in this case study. In particular the experience pointed to a variety of information gaps, issues from the perspective for the need for accessible, credible and evidence-based information. A number of lessons can be extrapolated from my patient journey. This includes: (1) the need for accessible health information that can be accessed from anywhere at any time (Kayser et al., 2015), (2) the need for access to second opinions within a timely period (Santiago et al., 2012), (3) the need for integration of healthcare information sources and personal medical information, such as medical records (Cimino et al., 2002), (4) the need for patient access to the most up-to-date information about diagnoses and treatment (Kushniruk, 2019), (5) the need for patient education information about long-term recovery (Wills \& Holmes-Rovner, 2003; Borycki et al., 2012) and (6) the need for easily accessible and usable decision aids for patients, families and health providers.

Mapping out the patient journey in this case study has indicated the need for more usable access to the right health information, resources, specialists and treatment (and the need for this in a timely manner). The role of e-health and consumer informatics for ensuring evidence-based patient choice can be critical, as this case study illustrated. This includes the need for widespread access by patients and physicians alike to evidencebased patient choice (EBPC), to achieve the following goal: "objective, unbiased information must be made available to the patient, and the patient must have the power and opportunity to choose" (Eysenbach \& Diepgen, 2001). Patient journey mapping can be a powerful mechanism for representing not only what a patient goes through, but also where processes can be improved and optimized for better health outcomes.

The importance of being able to concretely map out and analyze a patient's journey for complex healthcare cases, such as those associated with cancer, cannot be understated. In this paper we have described a complex case that involved a great number of issues and time sensitive decisions. We were able to visually describe this in a succinct and visual way using patient journey mapping. Despite a number of gaps and issues in the process by applying a human factors lens on the case and applying the best possible information available (both from healthcare providers and also from the publically available information over the WWW) a positive outcome was achieved.

Our current work involves using this type of mapping to study the patient journeys of multiple cancer patients to arrive at generalizable findings regarding where gaps and issues occur and where and how they can be rectified. There is considerable interest in patient-centred care and improving eHealth literacy (Monkman \& Kushniruk, 2015). However, achieving this will require methods that allow for pinpointing inefficiencies at all transitions of care, potential safety issues and problems that would 
preclude a positive clinical outcome. It is hoped that the case study provided in this paper illustrates the potential power of applying a patient journey mapping approach to both chronicling investigative details of patient journeys and to identifying specific areas where quality improvements could be made.

\section{Conclusion}

This paper has presented a case study along with an illustration of how patient journey mapping can be used to depict multiple perspectives and views of complex patient cases over time. The approach can be used to show both the efficiencies and gaps in care processes in a succinct and visual way that can then be used to help to investigate, understand and improve healthcare processes. The approach described in this paper is being used as the basis for a larger scale empirical study of cancer patient journeys, but can be generalized to a wide range of healthcare contexts and problems. In our future work we plan to develop patient journey maps for multiple individual patients and to further develop methods for generalizing individual patient journey maps to help identify critical issues in healthcare.

\section{Author Statement}

The authors declare that they have no conflict of interest.

\section{Acknowledgements}

The authors would like to thank Evangeline Wagner for her work in editing and formatting the final copy of this paper.

\section{ORCIID}

Andre W. Kushniruk (D) https://orcid.org/0000-0002-2557-9288

Elizabeth M. Borycki (iD https://orcid.org/0000-0003-0928-8867

Avi Parush (iD https://orcid.org/0000-0003-4435-8576

\section{References}

Borycki, E. M., Househ, F. M. S., Kushniruk, A. W., Nohr, C., \& Takeda, H. (2012). Empowering patients: Making health information and systems safer for patients and the public. Yearbook of Medical Informatics, 7(1), 56-64.

Canadian Cancer Society. (2020). Survival statistics for oral cancer. Retrieved From https://www.cancer.ca/en/cancer-information/cancer-type/oral/prognosis-andsurvival/survival-statistics/?region=on

Cimino, J. J., Patel, V. L., \& Kushniruk, A. W. (2002). The patient clinical information system (PatCIS): Technical solutions for and experience with giving patients access to their electronic medical records. International Journal of Medical Informatics, 68(1/3), 113-127.

Eysenbach, G., \& Diepgen, T. L. (2001). The role of e-health and consumer health 
informatics for evidence-based patient choice in the 21 st century. Clinics in Dermatology, 19(1), 11-17.

Eysenbach, G., \& Köhler, C. (2002). How do consumers search for and appraise health information on the world wide web? Qualitative study using focus groups, usability tests, and in-depth interviews. BMJ, 324: 573.

Kayser, L., Kushniruk, A., Osborne, R. H., Norgaard, O., \& Turner, P. (2015). Enhancing the effectiveness of consumer-focused health information technology systems through eHealth literacy: a framework for understanding users' needs. JMIR Human Factors, 2(1): e9.

Kushniruk, A. (2019). The importance of health information on the Internet: How it saved my life and how it can save yours. Journal of Medical Internet Research, 21(10): e16690.

Monkman, H., \& Kushniruk, A. W. (2015). eHealth literacy issues, constructs, models, and methods for health information technology design and evaluation. Knowledge Management \& E-Learning, 7(4), 541-549.

Parush, A. (2019). Designing health information for mutual empowerment in the joint journey of patients and healthcare professionals. Studies in Health Technology and Informatics, 257, 341-345.

Parush, A., Levin, M. L., \& Campbell, C. (2014). Dual patient-healthcare provider experience mapping and implications for information technology deployment and clinic layout. In V. Duffy \& N. Lightner (Eds.), Advances in Human Aspects of Healthcare (pp. 389-396). Boca Raton, FL: CRC Press.

Santiago, T. C., Jenkins, J. J., Pedrosa, F., Billups, C., Quintana, Y., Ribeiro, R. C., \& Qaddoumi, I. (2012). Improving the histopathologic diagnosis of pediatric malignancies in a low-resource setting by combining focused training and telepathology strategies. Pediatric Blood \& Cancer, 59(2), 221-225.

Wills, C. E., \& Holmes-Rovner, M. (2003). Patient comprehension of information for shared treatment decision making: State of the art and future directions. Patient Education and Eounseling, 50(3), 285-290.

Zhang, H., Dziegielewski, P. T., Biron, V. L., Szudek, J., Al-Qahatani, K. H., O’Connell, D. A., \& Seikaly, H. (2013). Survival outcomes of patients with advanced oral cavity squamous cell carcinoma treated with multimodal therapy: A multi-institutional analysis. Journal of Otolaryngology-Head \& Neck Surgery, 42: 30. 